Int. J. Dev. Biol. 50: 277-287 (2006)

doi: $10.1387 / \mathrm{ijdb} .052052 \mathrm{am}$

\title{
Morphodynamics of phyllotaxis
}

\author{
ALEXANDER G. MALYGIN* \\ Bakh Institute of Biochemistry, Moscow, and Institute of Cytology and Genetics SB RAS, Novosibirsk, Russia
}

\begin{abstract}
A morphodynamic model for phyllotaxis based upon an axiomatic approach is presented. We show that the helical forms of alternate phyllotaxis can be derived from the assumption of the rudiment's growth and movement on the cylindrical embryo surface in the absence of a longitudinal displacement. This leads to the repeating transition of tetragonal packaging of the rudiments into hexagonal packaging and vice versa. Under these conditions, sequences of rudiments produce either left-handed or right-handed helices, the number of which at the circumference of the cylinder corresponds to adjacent numbers of the Fibonacci series. Cross-opposite phyllotaxis forms are defined as superior with respect to the alternate ones, and verticillate phyllotaxis forms as superior with respect to the cross-opposite ones. Different phyllotaxis forms can be interpreted as a result of stretching of crystalline structures of the embryo formed by dense packing of rudiments. The superior phyllotaxis forms can be considered as the additive summation of lower forms. Morphodynamic mechanisms underlying the formation of multiple forms of helical phyllotaxis are discussed.
\end{abstract}

KEY WORDS: morphogenesis, phyllotaxis, helical form, polymorphism, Fibonacci series

\section{Mechanism of formation of the helical structures of alternate phyllotaxis}

Alternate phyllotaxis describes positions of leaves or other plant organs along the shoot axis in the plants that have just one organ in shoot nodes. This form of phyllotaxis exists either in the opposite (biserial) or the helical form. In the biserial alternate form, organs located at adjacent nodes (along the shoot axis) are situated opposite to each other. Therefore, the entire system has one plane of symmetry passing through its axis. In plants with alternate helical phyllotaxis, the location of organs along the shoot axis is helical (Fedorov et al., 1956). Helical forms of alternate phyllotaxis are the most common in the plant world. Specifically, such helical forms are typical for the oak (Quercus robur L.), cottonwood (Populus L.), alder (Alnus Mill.), hazel (Corylus avellanaL.), raspberry (RubusidaeusL.), apple-tree (Malus Mill.), pear tree (Pyrus L.), sunflower (Helianthus annuus L.), cabbage (Bras-sica oleracea L.), rose bud (Rosa L.), camomile heads (Matricaria L.), etc. Helical phyllotaxis may be characterized by either left-handed or right-handed genetic helices, i.e., spiral lines consecutively connecting plant organs, located next to each other along the axis of a stem or a shoot. In this situation, the line connecting the organs with the lower divergence angle is identified as the main genetic helix. The biserial form may be viewed as the limiting case of the helical form, when the angle of divergence between the adjacent organs is $180^{\circ}$. Such a form has a qualita- tive difference: specifically, left-handed and right-handed genetic helices in the biserial form are equivalent and the concept of the main genetic helix is no longer applicable. The biserial form is typical for grasses (Gramineae), elms (Ulmaceae), blueflags (Iridaceae), Amaryllidaceae and some other groups.

Helical forms of phyllotaxis obey strict numeric rules (Urmantsev, 1968). These rules are seen most clearly in the location of seeds in Compositae: the location of seeds repeats the helical position of leaves on the shortened axis. The essence of these rules is demonstrated by a deconvolution of the cylindrical surface (Fig. $1 \mathrm{~A}$ ) of the axial stem (Fig. 1B) of the spruce cone (Fig. 1C). As follows from the figure, adjacent traces of scales produce three types of parallel lines that are going around the cylindrical surface of the cone surface with different slopes. Such lines are referred to as parastichies. If we draw a circle of cylinder 00 ' around the center of an arbitrary trace on the convolution, the straight lines corresponding to parastichies with different slopes (Fig. 1A), this will yield triangles $0 \mathrm{~A} \mathrm{O}^{\prime}$ and $0 \mathrm{~B} \mathrm{O}^{\prime}$ with the circumference line of the cylinder $00^{\prime}$. The number of parastichies with similar slopes that intersect the lateral sides coincides with adjacent terms of the Fibonacci series: $1 ; 1 ; 2 ; 3 ; 5 ; 8 ; 13$, etc. (Each next term of this series, as can be easily seen, is the result of the summation of two previous terms.) For the case shown in Fig. 1A, the corresponding numbers are 3 (side 0'A), 5 (sides 0 'A and 0 B) and 8 (side $0^{\prime} B$ ).

The nature of numerical patterns associated with helical phyllotaxis has been the matter of studies for more than 400 
A

B

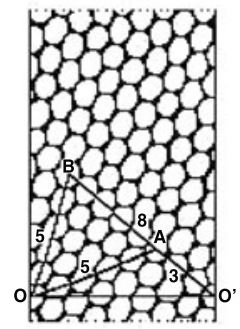

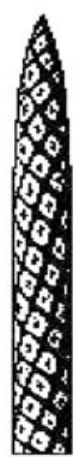

C

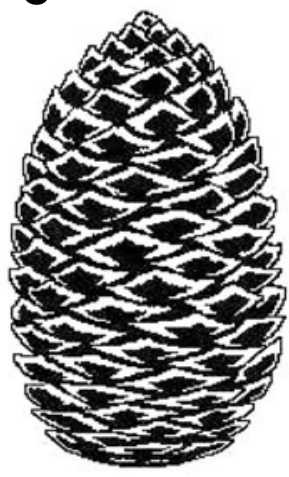

Fig. 1. Helical phyllotaxis of the spruce cone. (A) Imprint of deconvolution of the cylindrical surface of the core demonstrating the structure of the helical rudiment packaging intermediate between 3/5 Tetr and 5/8 Hex structures. (B) The axial portion cut from a spruce cone with clear parastichy helices on the cylindrical surface. (C) An overall view of the spruce cone with the main right-handed genetic helix.

years. Jean (1994) and Adler (Adler, 1974, 1975, 1977) are well known examiners of this problem. However, despite all of the success achieved in the modeling of helical phyllotaxis, there is no satisfactory explanation for the mathematical regularities of phyllotaxis (including non-helical forms) from a common theoretical platform. On the other hand, the unusual and enigmatic nature of these patterns gives a hope that their rational interpretation can become a key element in the development of a general theory of plant morphogenesis.

In our papers (Malygin, 1998, 2000-2002) we attempted to explain the morphodynamic origin of numeric patterns of the helical forms of alternate phyllotaxis on the basis of certain idealized views about the properties of plant rudiments and to use these patterns for explaining more complex forms of phyllotaxis.

It is obvious that the most convincing way to interpret the numerical patterns associated with alternate phyllotaxis would be to model the formation of the helical structure clearly demonstrating the appearance of new terms in the Fibonacci series by summation of the two previous ones. Here we demonstrate that by using an axiomatic approach based on the concept of an abstract "rudiment" and "embryo" endowed with some properties, we can build an adequate morphodynamic model. Specifically, the embryo will be referred to as a structure consisting of rudiments, which gives rise not only to the whole plant, but also to its individual organs (buds, leaves, central and lateral shoots, flow-

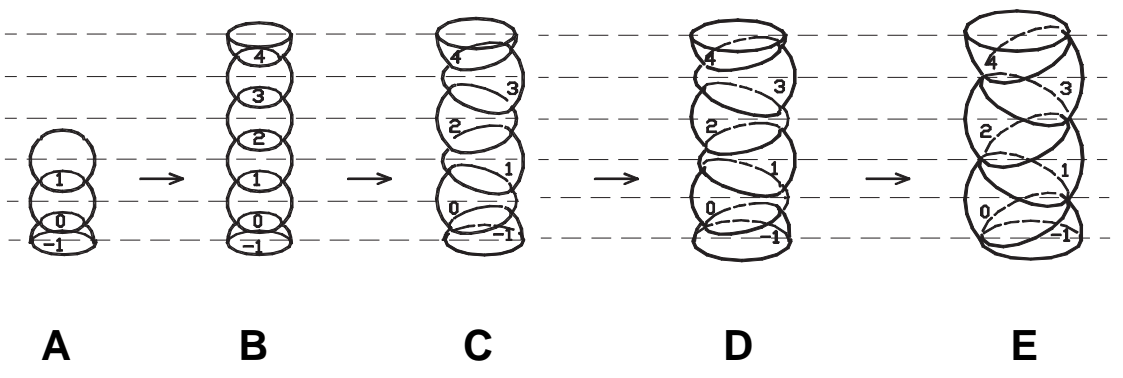

Fig. 2. Three-dimensional image of the early developmental stages (A-E) of a hypothetical embryo consisting of spherical rudiments (for explanation see text).

ers, roots, etc.).

The first property of the abstract rudiment is its ability to cleave and to produce embryos as a chain of the secondary rudiments, as shown in Fig. 2 A,B. Indeed, the early embryos of some grasses consist of individual metamers which look like spheres compressed along the vertical line (Kuperman, 1969a, b; Potapov, 1969). The second property is that the size of the rudiments increases proportionally to the growth duration. The thirdone is that while the rudiments' sizes become increased, their movement along the cylinder axis is restricted. Without discussing the nature of this limitation, we shall accept it as a feature intrinsic for plant rudiments. Taking these properties as axioms, we shall demonstrate that one mode of releasing the contradiction between increase in rudiment's size and disallowing their movement along the rudiment axis results in the helical forms of alternate phyllotaxis.

Our argument is formulated as follows. In Fig. 2, each rudiment corresponds to a certain level. These levels are indicated by horizontal dashed lines. During growth, the rudiments have a tendency to retain their spherical shape; since their movement in the direction of the embryo's axis is forbidden, they have to move in their level's plane, which is perpendicular to the axis of the embryo. As a result of the increased size of the rudiments, the vertical linear structure of the embryo becomes unstable. As a result, the movement of any level rudiment away from the axis (as shown on Fig. 2 B,C), will lead to the opposite movements of the rudiments situated on the neighboring levels. For greater clarity, on Fig. $2 \mathrm{C}-\mathrm{E}$, we show the movement of even rudiments to the left side and the odd rudiments to the right side.

In order to avoid problems associated with the increase of complexity, we will use planar graphic schemes. Correspondingly, we represent rudiments as circles, with their centers located at a certain level of the cylinder surface deconvolution; the corresponding level is indicated with a dashed horizontal line (Fig. 3). Fig, 2 illustrates the structural aspects of the joining rudiments. The sequence of three-dimensional structures in Fig. 2 corresponds to the sequence of planar structures in Fig. 3 A-E.

In Fig. 3 A,B, an increase in the number of circles reflects an increase in the number of rudiments due to their cleavage. The left and right edges of the deconvolutions result from the cutting of the lateral surface of the cylinders along their generatrices. Circles shown at the left and right edges of deconvolutions, in agreement with their right and left halves, respectively, are marked by identical numbers. In addition, the halves at the right edge of the deconvolution are marked with an apostrophe.

As shown in Fig. 3 C-E, the increase in the circles' diameter and their horizontal displacement results in the distribution of their centers between two diametrically opposite generatrices of the cylinder. For the sake of greater clarity, the centers of these circles are located on the generatrix corresponding to the cutting line of the lateral surface of the cylinder and the centers of odd circles are placed on the generatrix passing through the middle of this surface.

Quantitative relationships between spherical rudiments and their representations as flat circles can be illustrated by comparing Figs. 3,4 . Specifically, it can be seen that the width of 


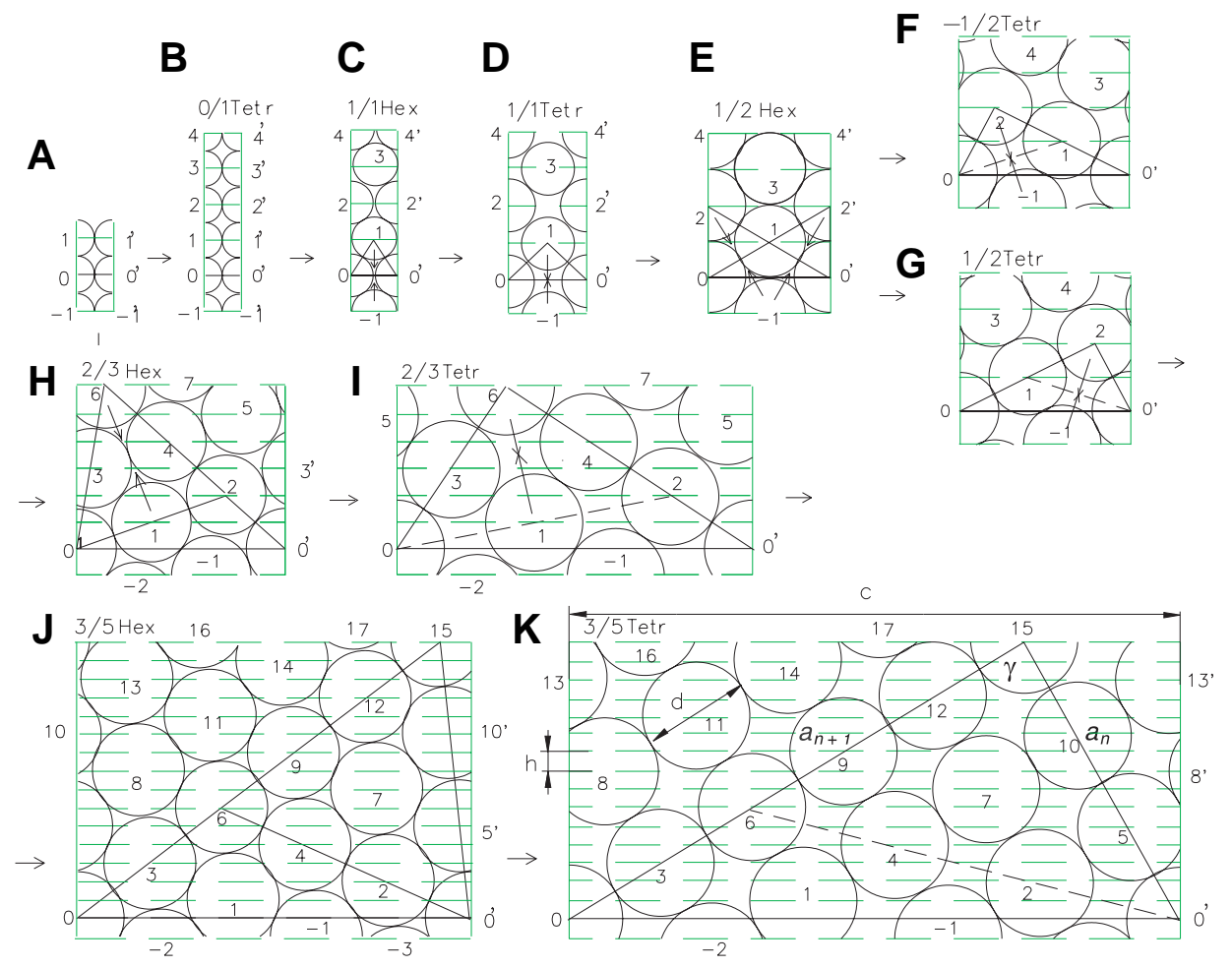

Fig. 3. Scheme of the development of helical forms of consecutive phyllotaxis represented as a sequence of deconvolutions of the lateral cylindrical surface of the growing embryo. Arrows on deconvolutions display the directions of the rudiments' horizontal movements (resulting from their size increase) and prohibited movements in the direction of embryonic axis (for explanation see text).

deconvolutions $c$ is determined by the circumference of the cylinder inscribed into horizontal elliptical projections of circles produced by mutual intersection of spheres. The diameter of circle $d$ is determined by the diameter of the largest circle, which is cut from the sphere by a plane touching the lateral surface of the cylinder. The distance between levels $h$ marked with dashed lines is determined by the distance between the horizontal planes, on which the centers of two sequentially numbered spheres are located.

We would like to demonstrate that, as the diameters of the circles increases, their packaging on the cylinder surface will eventually acquire the shape of helical structures and the number of observed parastichies will increase in accordance with the rule of the calculation of adjacent terms of the Fibonacci series. For demonstrating this, we use the circle numbering shown in Fig. 3.

In order to model the process of the formation of helical structures, we examine changes of triangle 00 ' 1 on deconvolutions of the cylinder (Fig. 3 B-E) during the growth of the circle diameter under the condition of the constancy of the distance $h$ between the levels. It can be easily seen that the circumference of the cylinder serves as a base for this triangle, while the lateral sides are produced by parastichies made from contacting circles. In our further discussion, triangles with these features will be referred to as characteristic triangles, while parastichies consisting of contacting circles will be referred to as contact parastichies.

As follows from Fig. $3 \mathrm{C}$, in the presence of dense hexagonal circle packaging, the characteristic triangle will be equilateral.
Therefore, the corresponding deconvolution can be briefly designated as $1 / 1 \mathrm{Hex}$, in which the fraction refers to the ratio of lateral sides of the triangle and the abbreviation Hex refers to the hexagonal packaging of the circles in deconvolution.

Figures 3 C-E illustrate the simplest case, when the number of contact parastichies in each of the two groups equals 1 . It can be easily seen from the position of the parastichies on deconvolution that they are weaving around the corresponding cylinder in the opposite directions along the helix. Under these conditions, the parastichies passing through points 012 ', etc., correspond to the right-handed genetic helix, while parastichies passing through the same points, but in a different sequence (0' 12 , etc.), comprise the left-handed genetic helix. Furthermore, the sequence of rudiments on genetic helices coincides with the original numbering of rudiments in the embryo shown in Figs. 2B,3B.

Since the circles comprising the contact parastichies come in contact with each other, the distances between the circle centers are equal to their diameters. Therefore, the conclusion can be drawn that the ratios between the lateral sides of the characteristic triangle will always be integral numbers.

The circle diameter in Fig. 3B is equal to the length of the cylinder's circuit and may be considered as the simplest contact parastichy with a zero slope to the horizontal line. This horizontal parastichy can be viewed as a degenerate characteristic triangle, with one side equal to zero. In view of the tetragonal placement of the circles, the deconvolution in Fig. $3 \mathrm{~B}$ by analogy with the deconvolution in Fig. $3 \mathrm{C}$ can be referred to as 0/1 Tetr.

An increase in the width of deconvolution due to the increase in circle diameter in the presence of a constant distance $h$ between circle centers along the axis (Fig. $3 \mathrm{C}$ ) is only possible if the contacts between the opposite points of circles on the horizontal parastichy $00^{\prime}$ are broken (Fig. $3 \mathrm{C}, \mathrm{D}$ ). This horizontal parastichy is then transformed from a contact to a noncontact parastichy.

An increase in the diameter of the circles and the transition of hexagonal circle packaging (Fig. 3 C,D) into tetragonal packaging transform an equilateral triangle into a right isosceles triangle. In this situation, the tetragonal packaging in Fig. 3D will be turned $45^{\circ}$ relative to the tetragonal packaging shown in Fig. $3 B$. By analogy with the previous cases, the deconvolution in Fig. 3D can be described as $1 / 1$ Tetr, where the fraction illustrates the ratio of the sides of the characteristic triangle, while the abbreviation Tetr refers to the ordered tetragonal packaging of circles on the deconvolution.

Similarly, the tetragonal packaging (Fig. 3D) will again be 
transformed into the right hexagonal packaging (Fig. 3E), while the angle of the isosceles characteristic triangle 010 ' opposite to the base will increase from $90^{\circ}$ to $120^{\circ}$. As a result, the ordered hexagonal packaging of circles will be rotated to $90^{\circ}$ relatively to the original one in the plane of deconvolution (Fig. $3 \mathrm{C}$ ). In this case, a group of two new parastichies appears alongside with two contact parastichies; these new parastichies will be referred to as the orthostichy due to their orthogonal position relative to the cylinder circumference. One of these orthostichy coincides with the lateral edges of deconvolution and passes through points 02 4 , etc. and $0^{\prime} 2^{\prime}$ 4', etc., while the second orthostichy passes through the central circles of deconvolution $-1 ; 1 ; 3$, etc.

Now, let us construct equilateral triangles 021 and 0 ' $12^{\prime}$ on each of the lateral sides of the isosceles characteristic triangle 0 $10^{\prime}$ (Fig. 3E). It can be seen that, in combination with the initial isosceles triangle, they will form two equal right mirrorly symmetric triangles ( $02^{\prime} 0^{\prime}$ and $\left.020^{\prime}\right)$. Because of the ordered orthogonal packaging, the sides of the two new triangles will match the lateral contact orthostichy and the corresponding contact parastichies coinciding with the left or right genetic helices. Therefore, these new triangles will be the characteristic triangles. Because they are equilateral, the smaller lateral side 02 of a new characteristic triangle is equal to the lateral side 01 of the initial isosceles triangle and their bigger side 0 ' 2 can be calculated as the sum of two lateral sides 01 and 0 ' 1 of the same triangle. Since the lateral sides of the triangles are located on the contact parastichies, their lengths can be expressed in terms of the integer number of circle diameters. It is easy to see that the length of the smaller lateral side of the new characteristic triangle will be equal to one

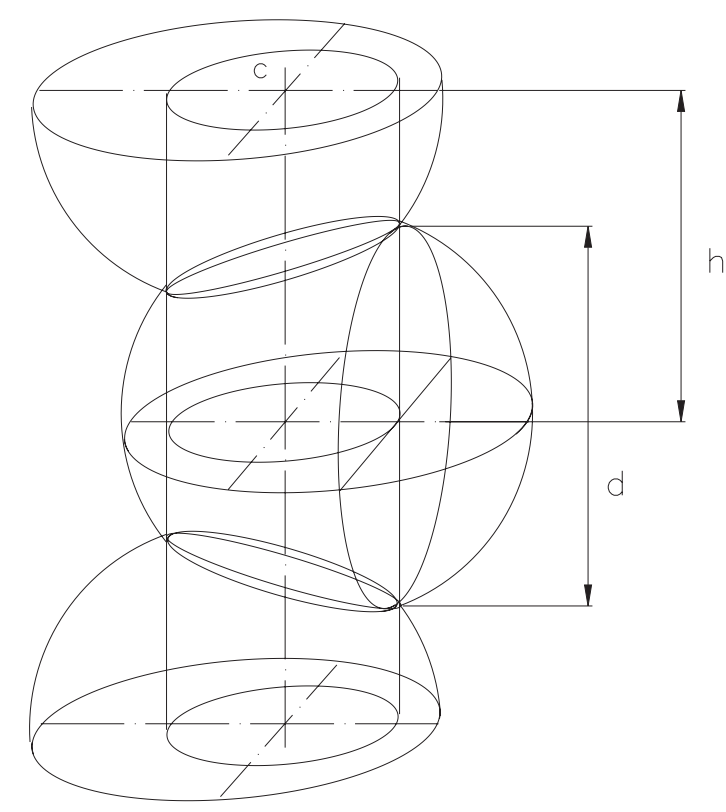

Fig. 4. Detailed scheme of a mutual intersection of spherical rudiments in embryos (see Fig. 2); $c$, the circumference of the cylinder corresponding to the width of deconvolution shown in Fig. 3; d, the diameter of the largest circle cut from the sphere by the plane touching cylindrical surface corresponding to the diameters of the circles equivalent to rudiments on deconvolutions (Fig. 3); $h$, the distance between the adjacent levels, at which spherical rudiments and the corresponding circles are shown on deconvolutions of Fig. 3. diameter, while the length of its bigger lateral side will consist of two diameters. In other words, it will be expressed by $d+d=2 d$, where $d$ is the circle diameter. On the basis of the properties of new characteristic triangles, the deconvolution shown in Fig. 3E will be described as $1 / 2 \mathrm{Hex}$.

As can be seen from the structure of deconvolutions from $1 / 1$ Hex to $1 / 2$ Hex (Fig. 3 C-E), the even rudiments of the corresponding embryos will be turned on the cylinder circumference by $180^{\circ}$ relative to the odd ones. As a consequence, all rudiments will be located in one vertical plane, which represents the only plane of the embryo symmetry. This means that the considered embryos correspond to two-column phyllotaxis. In view of the values of the angles in the characteristic triangles listed above (Fig. 3 B-E), we can easily demonstrate that the cylinder circumference $c$ corresponding to the width of deconvolution and circle diameter $d$ may be expressed through the distance between the levels $h$. Specifically, for Fig. 3B: $c=h, d=h$; for Fig. 3C: $c=2 h / \sqrt{ } 3, d=2 h / \sqrt{ } 3$, for Fig. 3D: $c=2 h, d=h \sqrt{ } 2$; and for Fig. 3E: $c=2 h \sqrt{ } 3, d=2 h$. These parameters unambiguously determine the diameter of the spherical rudiments and circles of their mutual intersection. Also, they determine the value of the horizontal displacement of the rudiments relative to each other (Fig. 4).

As can be seen in Fig. 3E, further increase in the circle diameter on the deconvolution and the corresponding increase in the deconvolution width due to the movement of the circles toward each other in the vertical direction is exhausted at this stage of development. Subsequent development of the structure may take place only through the break of contacts between circles located on one of the two inclined parastichies.

As shown in Fig. 3 F,G, the rupture of contacts on one or two parastichies results in the loss of their equivalence and, correspondingly, in the loss of the equivalence of genetic helices coinciding with them. Then, the parastichy, which retains the contacts with the adjacent rudiments having the minimal angle of divergence along the cylinder circumference, acquires the status of the main genetic helix.

Since the original parastichies are equivalent to each other and the system is in an unstable condition, the interaction which determines the choice of contact breakage on one or two parastichies, can be rather weak. In other words, at this stage, there can be an effective outside regulatory signal that will affect the selection of the sign of the main genetic helix of the embryo.

Furthermore, a loss in the equivalence of the genetic helices is accompanied by a loss of a symmetry plane and the change of phyllotaxis pattern from biserial to the alternate helical one. It is obvious that the embryos of grasses and other species with twocolumn forms of phyllotaxis mentioned earlier in this paper do not pass beyond the developmental stage shown on Fig. 3E.

Let us examine in more detail the consequences of the breakage of contacts in the parastichies passing through centers 0' 12 (Fig. $3 \mathrm{E}-\mathrm{G}$ ). In this situation, the right-handed (positive) genetic helix coinciding with the opposite parastichy and passing through centers 012 ' will be transformed into the main genetic helix and two orthostichy passing through centers 024 , etc. and -113 , etc., will lose their vertical position and will be transformed into the usual contact parastichy. As a result, out of two characteristic triangles produced by the intersection of parastichy and orthostichy in points 2 and 2', only one triangle produced by the intersection of the inclined parastichies and the former orthostichy in point 2 
A

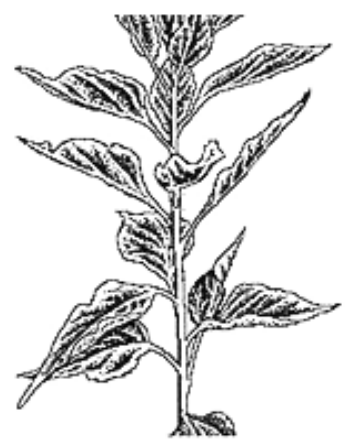

B

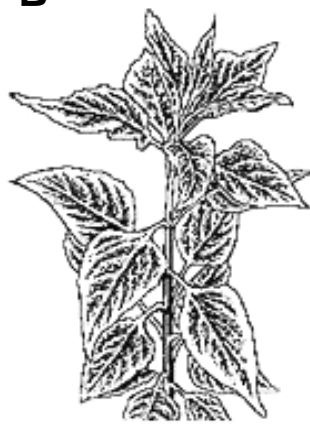

C

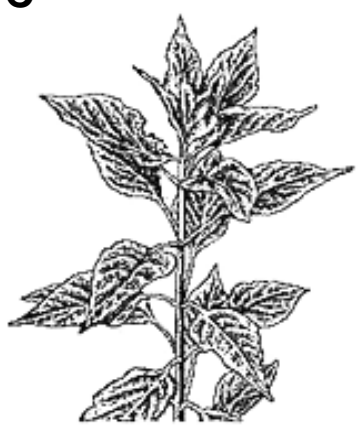

D

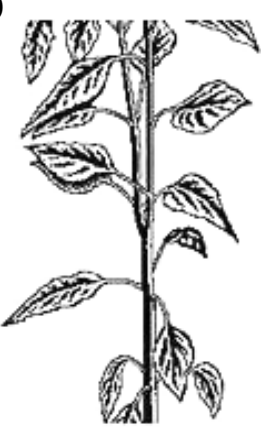

Fig. 5. Phyllotaxis forms in the Jerusalem artichoke. (A) Alternate helical, (B) cross-opposite, (C) three-leaved verticillate, (D) three-leaved verticillate at the base an cleaved into cross-opposite (left) and alternate helical forms (right). Found near the village Komarovka, Ryazan' district.

will remain. Because of the ratio between the sides of this triangle and the tetragonal nature of circle packaging, we define deconvolution in Fig. 2G as 1/2 Tetr.

A breakage of the parastichies passing through centers $012^{\prime}$ (Fig. $3 \mathrm{E}, \mathrm{F}$ ) will result in tetragonal packaging of the circles. The tetragonal packaging has a left-handed (negative) genetic helix and characteristic triangle with mirror symmetry. This is described as $-1 / 2$ Tetr.

It should be pointed out that the right-handed (positive) and left-handed (negative) helices in terms of their three-dimensional characteristics are respectively equivalent to the right-handed and left-handed screwthreads.

As the circle diameters and, correspondingly, the width of deconvolution increases, the tetragonal packaging transforms again into the ordered hexagonal packaging (Fig. $3 \mathrm{H}$ ). As a result, the angle at apex 2 of the characteristic triangle will be equal $120^{\circ}$ and the third group of contact parastichies will appear. If, just as in the previous case, we construct an equilateral triangle 062 on a bigger lateral side of the characteristic triangle, its combination with the characteristic triangle will produce a new characteristic triangle 060 '. Then, the smaller left side of the new characteristic triangle will be produced by the parastichies of the new group and its larger side (i.e., the right side) will be formed by the extension of the smaller side of the previous characteristic triangle 060 0'. Because the equiangular triangle 062 is also equilateral, the smaller side of the new characteristic triangle will be equal to the larger side of the previous triangle and the larger side 0 ' 6 will be equal to the sum of the larger and smaller sides of the preceding triangle. Since parastichies of all three groups are in contact, the length of the larger side of new characteristic triangle will be expressed as $d+2 d=3 d$. Therefore, deconvolution in Fig. $3 \mathrm{H}$ may be designated as 2/3 Hex.

Since the circle diameter and the deconvolution width will continue to increase, the structure of circle packaging will also continue to change. As in the previous case, this change is possible only because of the broken contacts between the circles on the contact parastichies. However, in this case, the situation is different, because nonsymmetrical positioning of the groups of contact parastichies on the deconvolution will unambiguously determine parastichies with the contacts between circles to be broken.

Since the parastichies of each group either match or are parallel to one of the sides of 026 triangle, the break of contacts of the parastichies of one group will increase the corresponding side of this triangle, as compared with the other two sides and lead to its transformation from an equilateral triangle into an isosceles triangle with the angle between the equal sides exceeding $60^{\circ}$. It is easy to see that the movement of the apices of triangle 026 due to the identical growth of all rudiments may transform this triangle from equilateral to isosceles one only if the contacts between rudiments on side 02 are broken. This side has a minimal slope to the horizontal (Fig. $3 \mathrm{H}, \mathrm{I}$ ) and, correspondingly, the angle at apex 6 will increase. In other words, the contacts between circles will be broken on the parastichies matching with the side 02 , which is the main genetic helix. At the same time, two other groups of parastichies will continue to keep contacts.

As a result of the increase in the circle diameter and the rupture of contacts between the circles on side 02 , the packaging passes again through the tetragonal structure (Fig. 3I) and returns to the hexagonal one (Fig. 3J), but in a different form. By constructing equilateral triangle $6150^{\prime}$ on the larger side of the preceding characteristic triangle 060 ' (Fig. 3J), we obtain a new characteristic triangle 0150 ' and find a new group of contact parastichies with a greater slope, as compared with the previous ones. Then, the smaller lateral side of a new characteristic triangle will be equal to the bigger lateral side of the preceding triangle and the larger lateral side will be equal to the sum of the lateral sides of the preceding triangle, which will be expressed by $2 d+3 d=5 d$. Therefore, this new deconvolution will be designated as $3 / 5 \mathrm{Hex}$.

After that, the hexagonal packaging will again be transformed into a tetragonal one via the procedure described earlier (Fig. 3K).

Transition from the hexagonal to tetragonal packaging of the circles and its returning back to the hexagonal one as a result of the increase in the circle diameters may, in principle, be repeated ad infinitum. It is clear that the rule of construction of the new characteristic triangle and the determination of the length of its bigger side as a sum of the sides of the previous triangle will be constant. It is easy to see that this rule corresponds with the recurrent formula for the calculation of each consecutive term of the Fibonacci series: $a_{n+2}=a_{n}+a_{n+1}$.

In other words, this scheme clearly demonstrates the correspondence between the morphodynamic mechanism responsible for the formation of the helical structure of the rudiments' arrangement and the Fibonacci series rule.

It is also easy to see that the number of parastichies parallel to one of the lateral sides of the characteristic triangle will be 

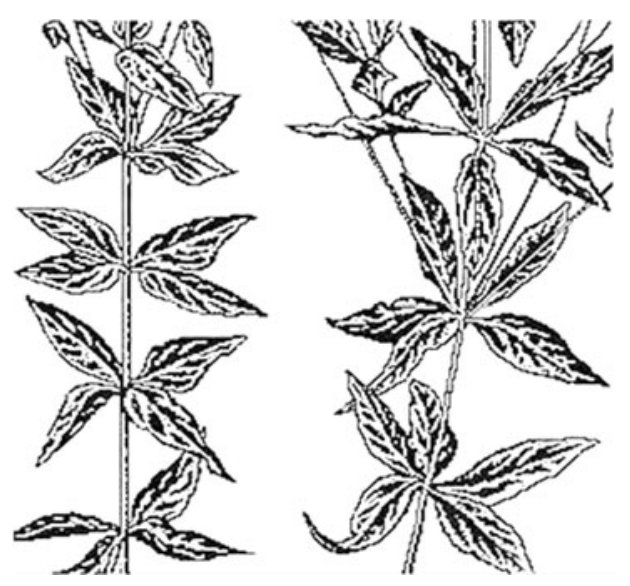

Fig. 6. Forms of phyllotaxis in the common loosestrife. (A) Verticillate four-leaved, (B) verticillate five-leaved (found in the Troparevo green zone, Moscow).

determined by the number of the circles center-to-center intervals on the opposite lateral side of the triangle. In other words, the number of parastichies in the groups of parastichies with opposite directions will be determined by the adjacent terms of the Fibonacci series.

The general quantitative description of the phillotaxis morphodynamic is given by the next two equations:

$$
\begin{aligned}
& c / d=\sqrt{a_{n}^{2}-2 a_{n} a_{n+1} \cos \gamma+a_{n+1}{ }^{2}} \\
& d^{2} / h c=\sin \gamma
\end{aligned}
$$

where $c$ is the length of embryo cycle, $d$ is the diameter of rudiments, $\gamma$ is the upper angle of characteristic triangle, $a_{n}$ and $a_{n+1}$ are the consecutive terms of the Fibonacci series corresponded to lateral sides of the characteristic triangle. The formulas are found by solution of the characteristic triangle (Fig. 3K).

Examination of the structure of deconvolutions in Fig. 3 demonstrates that the sign of the main genetic helix corresponds to the sign of the helical parastichy, on which the lateral sides of characteristic triangles are located, with the number of the centerto-center intervals being expressed by the term of the Fibonacci series with odd numbers. This opens up the possibility to derive the sign of the main genetic helix from the sign of the parastichy helix, in the cases when the main genetic helix is difficult to observe.

By using this scheme, we can interpret the development not only of embryos with a zero increase in the distance between the levels, but also of those embryos, in which the interlevels growth is slower than the radial one. Indeed, the axial increase in the size of the cylinder and the corresponding components of its radial increase can be explained by the proportional growth of the object. Since proportional growth in all directions does not change, the shape of the object is not changed (self-similarity is maintained). This kind of growth may be disregarded by considering the data presented in Fig. 3. This allows us to bring down all cases of the radial growth prevailing over the axial one to the above considered case of zero axial growth.

It is not difficult to see that the helical location of the scales on the spruce cone shown in Fig. 1B and its deconvolution in Fig. 1A correspond to the intermediate stage of the embryo transition from the shape illustrated by deconvolution 3/5 Tetr (Fig. 3K) to that illustrated by deconvolution $5 / 8 \mathrm{Hex}$ (not shown on Fig. 3 ).

If the helical form of phyllotaxis in fruit bodies is produced by the retention of the dense packaging of rudiments, the helical position of leaves observed in adult plants results from the axial extension of dense helical packaging of leaf rudiments in the embryo. Such an extension takes place when the axial growth of embryo occurs faster than its radial growth. At this time, the preexistent helical form of the rudiment positioning is fixed and, then, as rudiments are transformed into leaf plates, this pattern is transformed into the helical form of the alternate phyllotaxis observed in the plants described above.

In contrast, the discoid inflorescences with helical phyllotaxis such as those of a sunflower or a camomile may be produced by transformation of the cylindrical embryo through the conical form into a discoid.

Thus, the morphodynamic model constructed on the basis of an axiomatic approach explains the mathematical trends observed in helical forms of phyllotaxis.

\section{Theory of formation of the superior phyllotaxis forms}

The plants having one leaf in the stem or shoot nodes are referred to alternate phyllotaxis forms. Among the alternate forms, the opposite (biserial) and helical forms are distinguished when the angles between the leaves of adjacent nodes constitute $180^{\circ}$ and less than $180^{\circ}$, respectively. The structural properties of the alternate forms have already been considered in this paper.

The situation, when two leaves are located in one node and oppose each other by $180^{\circ}$, while the pairs of leaves from adjacent nodes are unfolded one with reference to another by $90^{\circ}$, is called cross-opposite phyllotaxis (Fedorov etal., 1956). The cross- opposite form has a second order symmetry axis and two planes of symmetry perpendicular to each other that pass through this axis. The cross-opposite phyllotaxis is inherent in the maple (AcerplatanoidesL.), ash (Fraxinus excelsior L.), lilac (Syringa vulgaris L.), horse chestnut (Aesculus hippocastanum L.),
Fig. 7. A sequence of three-dimensional structures (A-D) demonstrating the generation of a crystal-like dense packing of rudiments in the embryo, from which the cross-opposite phyllotaxis form is generated. 

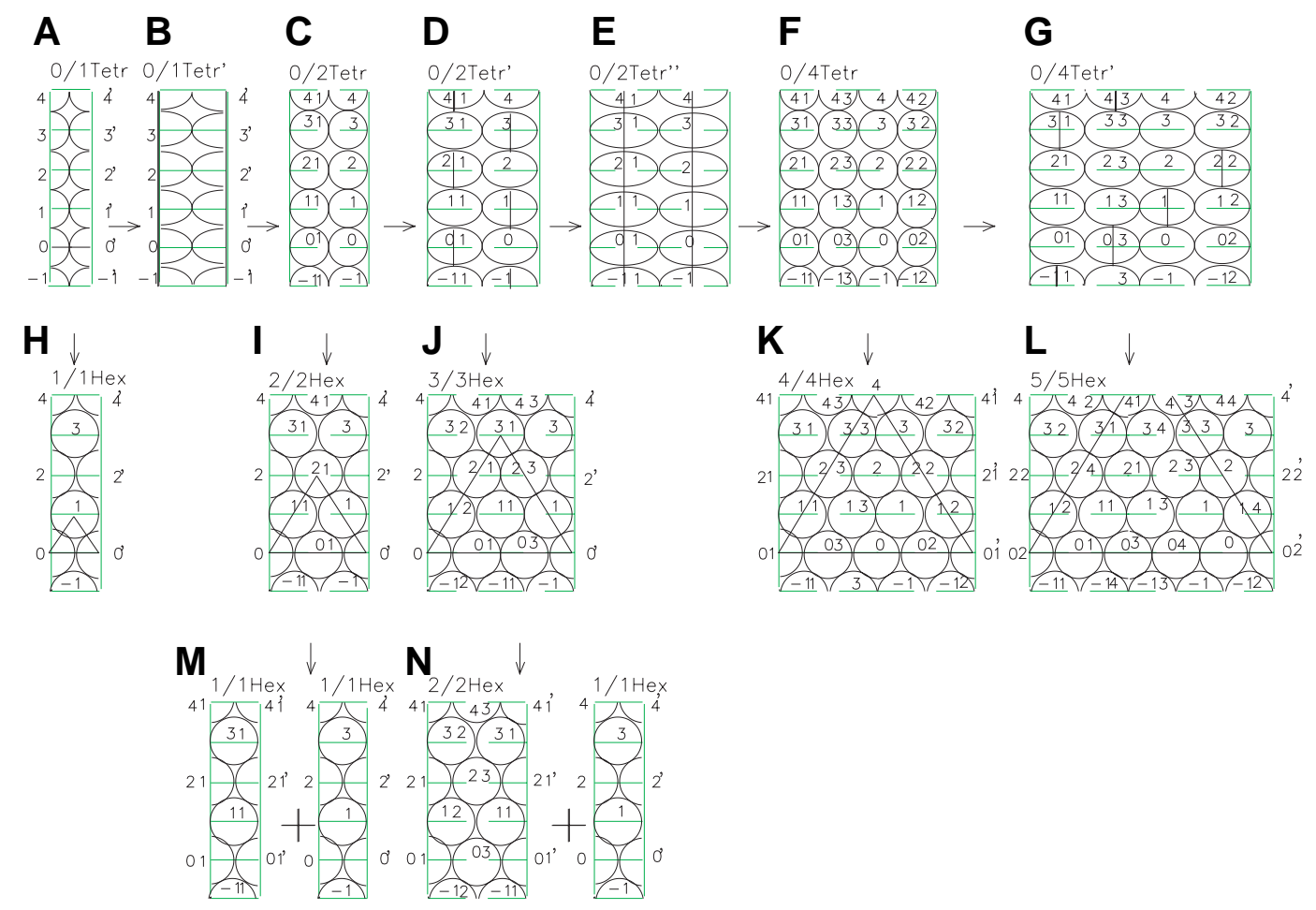

Fig. 8. Sequences of developments of the lateral surface of embryos demonstrating the mechanism of generation of cross-opposite and verticillate phyllotaxis. Numerals with hatches designate the halves of rudiments, located to the left of the dissection line of developments. The first and second numerals designate the sequence of the rudiments generation as a result of cleavage along the horizontal and vertical planes, respectively. The rudiment generated as a result of cleavage from the left half of the initial rudiments retains the ordinal number of the initial rudiment, while in the rudiment generated from the right half of the initial rudiment, the second numeral is substituted for a new, previously not used.

nettle (Urtica urens L.), hemp (Cannabis sativa L.), mint (Mentha arvensis L.) and many other plants.

In plants with verticillate phyllotaxis, three or more leaves are located in the same node. These leaves divide the stem circumference in equal parts. The verticillate forms have a symmetry axis, which order is determined by the number of leaves in a verticil and the corresponding number of symmetry planes. When three, four, or five leaves are located in the node, the angle between the neighboring leaves is $120^{\circ}, 90^{\circ}$, or $72^{\circ}$, respectively, etc. The leaves from the adjacent nodes are displaced one to another by a half of angle between the neighboring leaves in the node. As a result, the leaves of each node are located in gaps between the leaves of the upper and lower neighboring nodes. The arrangement of leaves in the nodes of branches of the oleander (Nerium oleanderL.) or of shoots around the stem of the shavegrass (Equisetumsylvaticum L.) can serve as examples of verticillate phyllotaxis.

It can easily be seen that the cross-opposite phyllotaxis forms can be considered as superior with reference to the alternate forms, since they have two leaves in the nodes, rather than one, as in alternate forms. The verticillate forms are, in turn, superior with reference to the cross-opposite forms, since they contain more than two leaves in their nodes (verticils). The verticillate forms with a greater number of leaves in the verticil are superior with reference to those containing a smaller number of leaves in the verticil.

The plants with verticillate phyllotaxis often have a variable number of leaves in the verticils on different branches. In some species, the number of leaves in the nodes can vary not only on different branches, but also within the limits of the same stem. Specifically, a variable number of shoots in the verticils is often observed in the shavegrass, as well as in the Jerusalem artichoke (Helianthus tuberosus L.) and loosestrife (Lysimachia vulgaris L.).

The Jerusalem artichoke and loosestrife convincingly prove by the variability of their forms the commonness of the mechanism underlying the formation of the alternate, opposite and verticillate phyllotaxis forms. The proof consists in that the Jerusalem artichoke plants with alternate helical (Fig. 5A), crossopposite (Fig. 5B), three-leaved verticillate (Fig. 5C) and even four-leaved verticillate phyllotaxis forms can grow simultaneously under the same conditions. Moreover, three phyllotaxis forms can sometimes be observed on the same Jerusalem artichoke plant, namely alternate helical, cross-opposite and three-leaved verticillate. The same is true for the loosestrife, in which, besides the crossopposite and three- and four-leaved verticillate forms (Fig. 6A), a five-leaved form can also occur (Fig. 6B). The phlox (Phlox paniculata L.) can also be referred to polymorphic species, in which cross-opposite and three- or four-leaved verticillate phyllotaxis forms may simultaneously occur.

How can the appearance of this diversity of phyllotaxis forms be explained on the basis of a common mechanism? Based on the pattern of symmetrical leaf arrangement in cross-opposite and verticillate forms, it can be proposed that in adult plants, these 
A

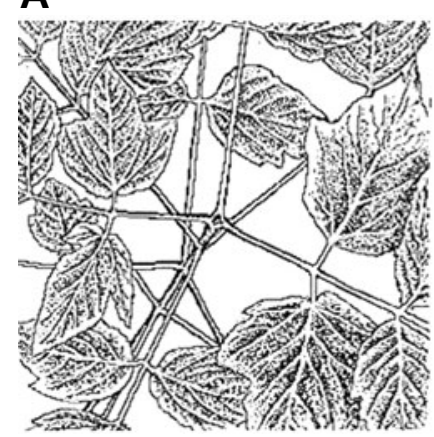

B

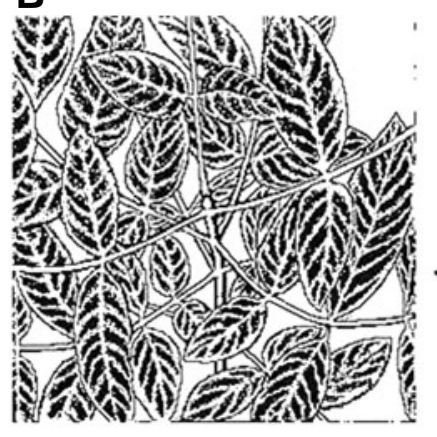

Fig. 9. Rare forms of three-leaved verticillate phyllotaxis in trees. (A) In the maple (found on Lenin Avenue in a square near the Institute of Biochemistry, Russian Academy of Sciences. (B) In the ash (found on Lenin Avenue in the square at the Institute of Biochemistry). (C) In the lilac (found in Komarovka village, Ryazan District and in Pushchino).

forms arise upon stretching of the embryo along the axis of its cylindrical surface formed by the crystal-like structure of dense packaging of rudiments, similar to the packaging of atoms in crystals. The simplest structures of this kind are shown in Fig. 7. In order to answer the question about the mechanism of appearance of crystal-like structures, let us look the initial stages of embryo cleavage into rudiments. In the case of alternate phyllotaxis, the cleavage of rudiments could proceed only in the axial direction, while the translocation of the rudiments along the embryo axis is forbidden. The controversy between the growth of the rudiments volume and a prohibition of their translocation in the axial direction is solved by their alternate translocation in the horizontal plane, that is, perpendicular to the embryo axis. However, this controversy can be solved also in another way, namely due to the cleavage and translocation of the rudiments in the horizontal plane. Now the vertical distance between the rudiments remains invariable, while the total volume of rudiments is increased because of their increased number.

What could such a kind of cleavage be due to? It is evident that the prohibition of the axial translocation should lead to compression of the rudiments from below and from above as a result of their growth. If the rudiments rigidity is great enough, the compression will enhance their horizontal dislocation. Meanwhile, if a rigidity is smaller, the compression can deform the rudiments into ellipsoids (Fig. 7 A,B) with their subsequent cleavage (Fig. 7 B,C) and displacement of newly formed rudiments around the axis with their dense packing (Fig. 7 C,D), similar to the packing of atoms in the crystalline grid. The cleavage of the rudiments in the direction of the long ellipsoid axis corresponds to Hertwig's rule for egg cleavage. Thus, the different behavior of rudiments leading to the formation either of the alternate or the higher phyllotaxis forms can be caused by the differences in their rigidity. The changes in the mechanical properties of the rudiments can be due to the changes in plant metabolism, either genetically or environmentally determined.

For simplification, the formation of embryos of the crossopposite and verticillate phyllotaxis forms is shown in Fig. 8 with the help of the developments of the embryo cylindrical surface. As seen, the structures of all the developments are composed of elementary fragments formed by the rudiments and designated as $0 / 1 \mathrm{Tetr}, 0 / 1 \mathrm{Tetr}$, or $1 / 1 \mathrm{Hex}$. In the full correspondence with the 3D figures from Fig. 7, the Fig. 8 A-C display how the

C

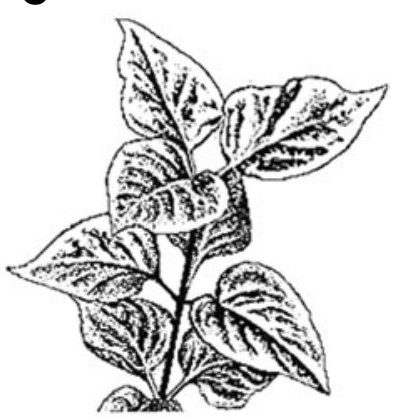

rudiments designated as circles are deformed into ellipses under the influence of the growth and how they are finally cleaved in halves. In some cases the cleavage of one rudiment at each level may be sufficient for dissipating the compression force experienced by the rudiments (Fig. 8 B,D,G), while in other cases the cleavage of two or more rudiments is necessary (Fig. $8 \mathrm{E}$ ). It is proposed that if the levels include more than one rudiment, the cleavage of a single one leads to the horizontal displacement of all the others from the same level and induces the transition of the embryo from an unstable tetragonal packaging structure (Fig. 8 $D, G)$ to a dense hexagonal structure (Fig. $8 \mathrm{~J}, \mathrm{~L}$ ). If all the rudiments are cleaving simultaneously (Fig. 8 B,E) this leads to their synchronous displacement at different levels and, as a result, an embryo with an unstable tetragonal structure of packing of rudiments is formed (Fig. 8 C,F).

If the rigidity of the rudiments in the tetragonal structure of packing is insufficient, the rudiments are cleaving as a result of growth (Fig. 8 B,D,E,G). However, if their rigidity is increased up to a certain limit, the different levels rudiments are displaced along the horizontal plane, forming thus more stable hexagonal structure (Fig. $8 \mathrm{H}-\mathrm{J}, \mathrm{K}$ ).

Therefore, if the rigidity of the rudiments at the initial developmental stages is sufficient, the embryo passes from unstable structure $0 / 1$ Tetr into the stable structure $1 / 1 \mathrm{Hex}$, as shown in Figs. $8 \mathrm{a}, 8 \mathrm{~h}$ and then in the helical structure of alternate phyllotaxis according to the scheme earlier considered (Malygin, 1998, 2001). This structure corresponds to the helical leaf arrangement in the Jerusalem artichoke (Fig. 5A). In the opposite case, the embryo changes the structure $0 / 1$ Tetr for a new structure $0 / 2$ Tetr as a result of a compression and cleavage of the rudiments (Fig. $8 \mathrm{~B}, \mathrm{C})$.

An alternative of the choice is then repeated for the embryo $0 /$

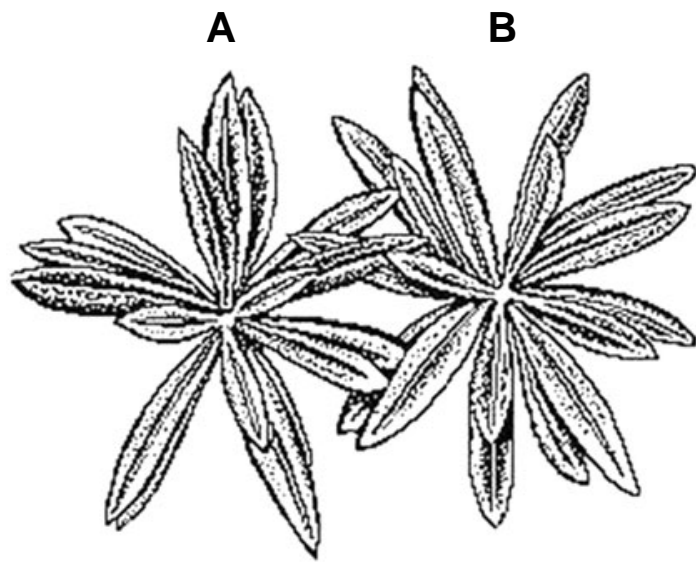

Fig. 10. Forms of verticillate phyllotaxis in the oleander. (A) Threeleaved, (B) four-leaved (found among the decorative plants at the Institute of Protein Synthesis, Russian Academy of Sciences, Pushchino). 
2 Tetr. When the rudiments reach a sufficient rigidity, unstable tetragonal structure of the embryo $0 / 2$ Tetr passes into the stable hexagonal structure 2/2 Hex (Fig. $8 \mathrm{C}, \mathrm{I}$ ). In the 3D form, this transition is shown in Fig. 7 C,D. It leads to the cross-opposite phyllotaxis form represented by a Jerusalem artichoke plant in Fig. 5B.

In the case of a smaller rigidity, one (Fig. $8 \mathrm{D}, \mathrm{J}$ ) or two (Fig. 8 $E, F)$ of each level rudiments cleave, as a function of their compression. The cleavage of one rudiment stimulates the generation of a solid hexagonal structure $3 / 3$ Hex corresponding to threeleaved verticillate phyllotaxis form, like in a Jerusalem artichoke plant shown in Fig.5c. The cleavage of two rudiments at each level leads to the generation of unstable hexagonal structure 0/4 Tetr (Fig. 8F).

Similar to the above described cases, an embryo with unstable tetragonal structure 0/4 Tetr can develop in one of two alternative directions. When the rudiments reach the necessary rigidity, the unstable structure of packaging $0 / 4$ Tetr passes in the stable structure 4/4 Hex (Fig. 8 F,K), which corresponds to a four-leaved verticillate phyllotaxis form, like in a loosestrife plant shown in Fig. $6 \mathrm{~A}$. Otherwise one or two each level rudiments are cleaved, as a function of the compression degree. The cleavage of one rudiment at each level stimulates transition to the stable hexagonal structure $5 / 5$ Hex (Fig. 8 G,L), which corresponds to the fiveleaved verticillate phyllotaxis form, like in a loosestrife plant shown in Fig. 6B. The cleavage of two or more rudiments leads to the superior verticillate phyllotaxis forms.

Since the maximal axial pressure should develop during the period of enhanced growth of rudiments (which corresponds usually to the formation of the middle part of plant stem), the greatest number of the rudiment cleavages and, correspondingly, the greatest number of the leaves or shoots in the verticils is usually observed in the middle part of a stem.

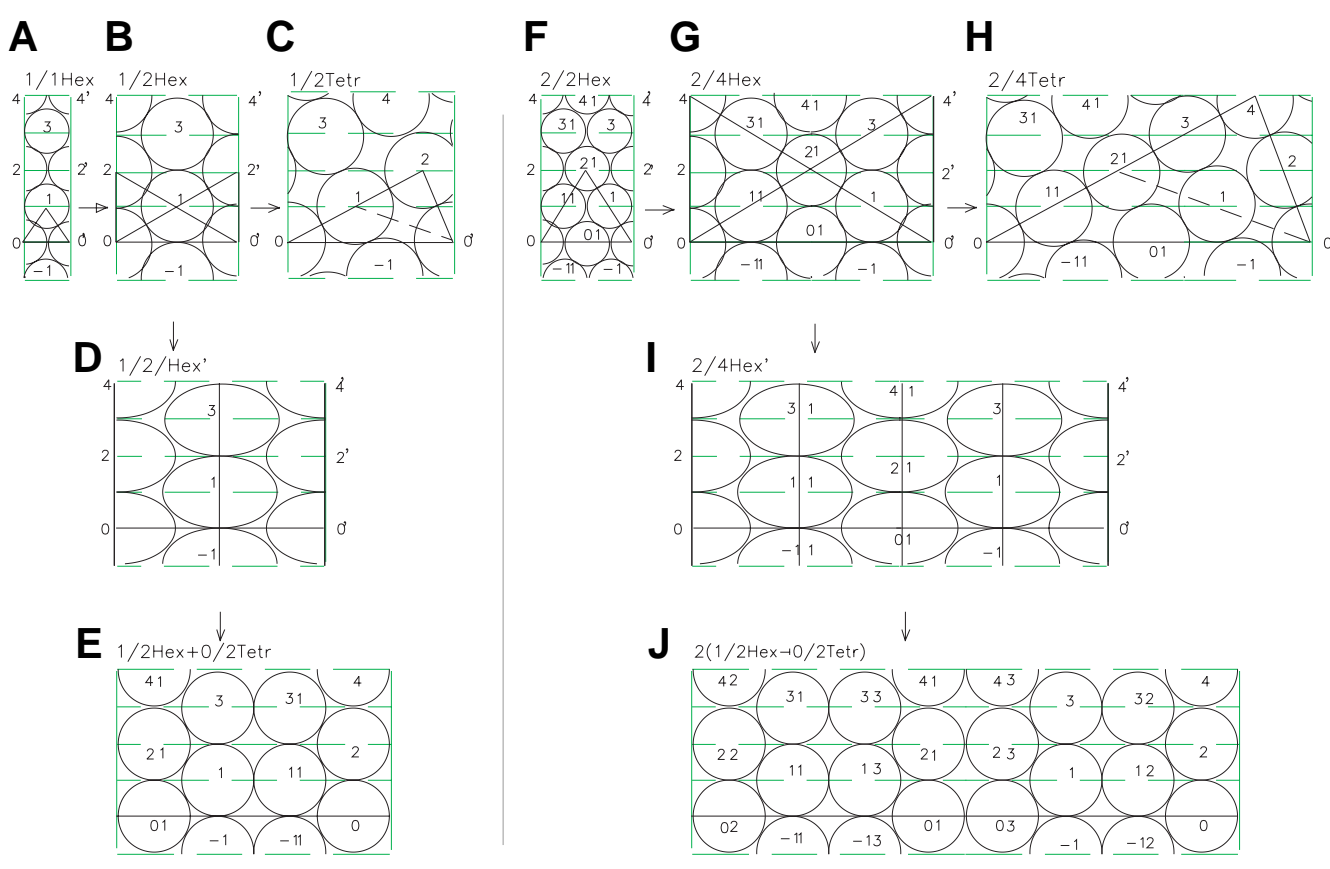

Fig. 11. Sequences of developments of the lateral surface of embryos demonstrating the alternative generation of hexagonal-tetragonal and the multiple helical forms of phyllotaxis (for explanations see text).
On the other hand, the transition from the opposite location of two first leaves in the seedlings of some species towards the alternate form of location of subsequent leaves in the adult plan can be explained by the loss of the rudiments' cleavage capacity due to their enhanced rigidity.

Therefore, such a mechanism explains the ambiguity of phyllotaxis in the conspecific plants by the variations in the rudiments cleavage capacity, which can be, in its turn, explained by the environmentally dependent variability of the elastic properties of cells walls during embryo formation.

The model of generation of the different phyllotaxis forms resembles the rearrangements of the minerals crystalline grid under the influence of external pressure (Zharov, 1988). The difference between plants and minerals is that in the first case the pressure is regulated by the internal causes.

The consequences from our theory are confirmed by following. For demonstrating that the phyllotaxis pattern is not a species specific qualitative feature but can be changed due to environmental factors (Fig. 8), we searched for the deviations in the phyllotaxis in trees usually characterized by stable phyllotaxis patterns. As a result, in addition to cross-opposite forms of phyllotaxis in the maple (Fig. 9A), ash (Fig. 9B) and lilac (Fig. 9C), three-leaved verticillate forms could be found. In these species, the transition from a cross-opposite form to the verticillate one takes place as an appearance of one or several shoots with three leaves in the verticils. The oleander can serve as an example of transition from the three-leaved verticillate (Fig. 10A) form to a four-leaved one (Fig. 10B).

Another conclusion from the theory is that the embryo of superior phyllotaxis forms can be considered as the result of additive summation of the embryos of lower forms. If this statement is true, the opposite process, namely a transition of the superior forms cleavage to that of the lower ones should take place under certain conditions. Thus, the cross-opposite phyllotaxis form will cleave in two alternate forms (Fig. 8 I,M), while the three-leaved verticillate form cleaves in the cross-opposite and alternate forms (Fig. $8 \mathrm{~J}, \mathrm{~N}$ ).

Actually, the cleavage of the branch with the opposite phyllotaxis in two shoots with alternate helical forms as a result of diagonal cut of the apex of the willow herb (Epilobium hirsutum L.) was described long ago (Snow and Snow, 1935), although at that time this result was difficult to interpret. Now its explanation based upon a scheme (Fig. $8 \mathrm{I}, \mathrm{M}$ ) looks as trivial.

Our concept is also confirmed by a branching of the Jerusalem artichoke verticillate stem in two shoots, one of which has a crossopposite phyllotaxis and another the helical form of alternate phyllotaxis (Fig. 5D). This phe- 
nomenon is explained by the scheme given in Fig. $8 \mathrm{~J}, \mathrm{~N}$.

\section{Hexagonal-tetragonal type of phyllotaxis}

The above discussion on the mechanisms of cross-opposite and verticillate phyllotaxis left unanswered the question of existence of the intermediate unstable tetragonal forms. Meanwhile, they are of a crucial significance for our concept. Since intermediate tetragonal forms are not recorded in adult plants during generation of the hexagonal forms of the rudiments packing, the direct confirmation of their existence is intricate. However, if the cleavage of the rudiments leading to the generation of tetragonal forms at the stages 0/1 Tetr, 0/2 Tetr, etc. (Fig. 8) takes place, it can also be realized at the stages $1 / 2 \mathrm{Hex}, 2 / 4 \mathrm{Hex}$, etc. (Fig. 11). In this case, the primary result of cleavage can be sustainable recorded in the hexagonal grid of the embryo, as was shown for the form 1/2 Hex (Fig. 11 B,G,I) and 2/4 Hex (Fig. 11 E,H,J) and be preserved in the adult plant. Since such a cleavage forms the linear vertical fragment of tetragonal grid, which is incorporated in the hexagonal grid, the resulting configuration of rudiments can be defined as a mixed hexagonal-tetragonal phyllotaxis.

The hexagonal-tetragonal arrangement of leaves on the stem is observed in the family Solanaceae. Specifically, it is typical for the strawberry tomato (Physalis alkekengi L.). As can be seen from Fig. 12A, the paired arrangement of the leaves in each node corresponds to the paired distribution of rudiments at the individual levels of cylindrical development (Fig.11I). It can be easily seen that the pair of the vertically adjacent leaves are unfolded to $180^{\circ}$ from each other, like the pairs of rudiments from the adjacent levels. Namely, the pair of rudiments 111 at the first level is turned by $180^{\circ}$ with reference to the pair of rudiments 010 at the zero level, the pair of rudiments 212 at the second level is turned by $180^{\circ}$ with reference to the pair of rudiments 111 at the first level, etc.

The hexagonal-tetragonal phyllotaxis form is often reflected in the arrangement of grains in the ear of Poaceae. In the bamboo (Bambusa L.), it is expressed as close paired lateral shoots in the culminating nodes. According to the theory, these pairs in the neighboring nodes are unfolded by $180^{\circ}$ with reference to each other.

If the cleavage of the rudiments is taking place in the embryo
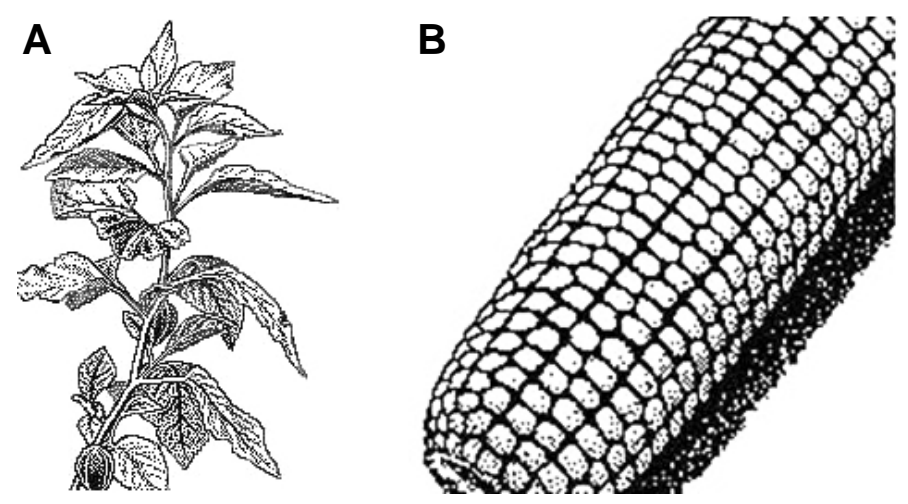

Fig. 12. Examples of the hexagonal-tetragonal phyllotaxis. (A) Leaf arrangement on the strawberry tomato stem. (B) Packing of grains in the corn (Zea mays L.) ear. with an obvious verticillate phyllotaxis, the packing is formed, in which the paired hexagonal-tetragonal elements are repeated many times. The distribution of grains in the corn (Zea mays L.) ear is an example (Fig. 12B). The number of repetitions of the hexagonal-tetragonal fragment on the ear varies from four to ten units.

Identification of specific mechanism underlying the hexagonaltetragonal distribution of organs on the stem not only allows us to define the corresponding type of phyllotaxis as a special one, but proves also the existence of intermediate unstable tetragonal forms during generation of the opposite and verticillate phyllotaxis forms.

\section{Generation of multiple forms of helical phyllotaxis}

In this section we consider the mechanism of generation of the multiple forms of the helical phyllotaxis which development look like duplicated, triplicated, etc. developments of a simple helical packing. The doubled forms are most frequent. Some of them occurring in the Norfold Island pine (Araucaria excelsa L.), stonecrop (Sedum elegance L.), Silphium L. and Podocarpus L. were described by Hirmer (1931). The duplicated form was studied in more detail in the cut-leaved teasel (Dipsacus laciniatus L.) (Snow, 1950).

What is the mechanism of generation of the multiple forms of helical phyllotaxis? One may see that they can arise as a result of coordinated growth of rudiments in two or more parallel embryos contacting during their development. In other words, the development of these forms can be a continuation of development of the opposite (Fig. 8I) and verticillate (Fig. $8 \mathrm{~J}-\mathrm{L}$ ) phyllotaxis forms according to the scheme of generation of simple helical forms (Malygin, 1998, 2001). It can be seen in Figs. $8 \mathrm{H}-\mathrm{L}, 11 \mathrm{~A}-\mathrm{F}$ that the difference between the simple and multiple forms of helical phyllotaxis consists in multiplication of the number of intercenter intervals on the lateral sides of characteristic triangles $020^{\prime}$ and $040^{\prime}$ in the embryos and, correspondingly, in multiplication of members of the normal Fibonacci sequence characterizing the number of parastichies in the simple helical phyllotaxis.

The initial stages of development of a simple (Fig. 11C) and duplicated (Fig.11F) forms from the alternate (Fig. $11 \mathrm{~A}, \mathrm{~B}$ ) and cross-opposite (Fig. $11 \mathrm{D}, \mathrm{E}$ ) ones, respectively, are compared on cylindrical developments (Fig. 11) as an alternative to the development of simple (Fig. 11 B,G,I) and duplicated (Fig. 11 E,H,J) forms of the mixed hexagonal-tetragonal phyllotaxis.

Since in the case of the multiple forms, two or more rudiments are located at the same level of the embryo, these forms contain also the corresponding number of genetic helices coiled in parallel. The sequences of rudiments 0112134 and -110112 are such helices(Fig. 11F). The independent nature of parallel genetic helices was experimentally proved by Loiseau (1954) who succeeded to decrease the number of leaf helices from three to two by destroying one young rudiment at the balsam (Impatiens royleiWalp.) apex.

The true multiple forms of helical phyllotaxis should be distinguished from the apparent ones. In the true multiple forms, unlike the apparent ones, the rudiments from different helices are located at the same levels. The apparent multiple forms arise as a result of axial or radial extension of one embryo with definitive helical phyllotaxis, rather than from parallel embryos. All the 
rudiments of this embryo are located successively on the single genetic helix and, hence, are arranged at the different levels.

When an embryo is extended either axially or radially, the proportionally growing rudiments preserve their physical contacts on the parastichia parallel to the longer or shorter sides of the characteristic triangle, respectively. Hence, these parastichia are perceived subjectively as independent helical structures. It is natural that the number of these helices be determined by the number of corresponding parastichia, i.e., the Fibonacci number, which corresponds to the number of rudiments on the opposite side of the characteristic triangle. It may well be that these apparent leaf helices were described by Plantefol (Plantefol, 1948; see also Sinnot, 1963) who studied parallel helices in plants.

The lesser occurrence of the multiple helical forms in nature, as compared to alternative hexagonal-tetragonal forms appears to be due to the fact that the substitution of the initial elasticity of the rudiments (essential for the formation of embryos with opposite, verticillate, or hexagonal-tetragonal phyllotaxis) by their rigidity (essential for the generation of multiple helical forms) requires more complex and, hence, less frequent genetic developmental program.

Note also that in addition to the opposite and verticillate forms, the mixed hexagonal-tetragonal forms can be subjected to a coiling. For example, a weak coiling of the hexagonal-tetragonal phyllotaxis is often observed in the corn ears. The existence of these forms can be provided by an early stage generation of an unstable equilibrium between a coiling of the cross-opposite or verticillate forms into a helical structure and the binary cleavage of rudiments.

Thus, our concept may promote in explaining the relationships between the morphodynamic of superior and lower phyllotaxis forms in different plants, in understanding the nature of polymorphism of phyllotaxis in conspecific plants and in describing a beforehand omitted hexagonal-tetragonal type of phyllotaxis.

\section{References}

ADLER, I. (1974) A Model of Contact Pressure in Phyllotaxis. J. Theor. Biol. 45: 179.
ADLER, I. (1975). A Model of Space Filling in Phyllotaxis. J. Theor.Biol. 53: 435444.

ADLER, I. (1977). The Consequences of Contact Pressue in Phyllotaxis. J. Theor. Biol. 65: 29-77.

FEDOROV, A.A., KIRPICHNIKOV, M.E. and ARTYUSHENKO, Z.T. (1956). Atlas po opisatel'noi morfologii vysshikh rastenii. List (Atlas on Descriptive Morphology of Higher Plants. The Leaf). Moscow: Izd. Akad. Nauk SSSR.

JEAN, R.V. (1994). Phyllotaxis. Cambridge Univ. Press.

KUPERMAN, F. M. (1969a). Physiology of Development, Growth and Organogenesis. Wheat. In Fiziologiya sel'skokhozyastvennykh rastenii (Physiology of Agricultural Plants). Moscow: Izd. Mosk. Univer. 4: 7-193.

KUPERMAN, F.M. (1969b). Physiology of Wheat Resistance. In Fiziologiya sel'skokhozyastvennykh rastenii (Physiology of Agricultural Plants), Moscow: Izd. Mosk. Univer. 4: 401-490.

LOISEAU, J.E. (1954). Suppression expèrimentale d'une Helice Foli-are chez. Impatiens royleiWalp. Compt. Rend. Acad. Sci. (Paris) 238: 149-151.

MALYGIN, A.G. (1998). Structural Theory of Phyllotaxis. I. Mechanisms of Formation of Helical Structures of Consecutive Phyllotaxis. Biofizika. 43: 335-342.

MALYGIN, A.G. (2000). Structural Theory of Phyllotaxis. II. Interrelation between Lower and Higher Forms of Phyllotaxis. Biofizika. 45: 1112-1118.

MALYGIN, A.G. (2001). Theory of Phyllotaxis. 1. Geometrical Model of the Formation of Helical Forms of Alternate Phyllotaxis. Ontogenez (Moscow). 32: 393400 .

MALYGIN, A.G. (2002). Theory of Phyllotaxis. 2. Crystallographic Interpretation of the Interpretation between Lower and Superior Phyllotaxis Forms. Ontogenez (Moscow). 33: 471-478.

PLANTEFOL, L. (1948). La theorie des helices foliaires multiples. Fon-daments d'une theorie phyllotaxique nouvelle. Paris: Masson.

POTAPOV, N.G. (1969). Mineral Nutrition of the Wheat. In Fiziologiya sel'skokhozyastvennykh rastenii Physiology of Agricultural Plants), Moscow: Izd. Mosk. Univer. 4: 242-292.

SINNOT, E. (1963), Morfogenez rastenii (Morphogenesis of Plants), Moscow: Inostr. Lit-ra: 181

SNOW, M.and SNOW, R. (1935). Experiments on Phyllotaxis. 3. Diagonal Splits through Decussate. Apices, Phil. Trans. Roy.Soc. B. 225: 63-95.

SNOW, R. (1950). Experiments on Bijugate Apices. Philos. Trans. Roy. Soc., B. 235: 291-310.

URMANTSEV, Y. A. (1968). The Golden Section. Priroda. 11: 33-40.

ZHAROV, A.A. (1988). Pressure, Khim. entsiklopediya (Chemical Encyclopedia), Moscow: Sov. Entsiklopediya. 1: 619-622. 